\title{
Free Vibration Analysis of L-Shaped Folded Thin Plates
}

\author{
Koji Sekinea,* \\ ${ }^{a}$ Field of Mechanical Engineering, Department of Creative Engineering, National Institute of Technology, Kushiro College \\ Email:sekine@kushiro-ct.ac.jp
}

\begin{abstract}
Free vibration analysis of L-shaped folded thin plates having various boundary conditions is presented. Vibration characteristics of the folded plates are analyzed by means of the Ritz method. Displacement functions satisfying the geometric boundary conditions are assumed in the form of double power series. The interconnection of plate elements of the folded plates is defined by translational and rotational coupling springs. The generalized eigenvalue problem, which is derived by means of minimizing the energy functional, is solved to determine the natural frequencies and mode shapes. The accuracy and validity of the present solutions are demonstrated through convergence studies and comparisons with the results from the literature and FEM (finite element method) analysis solutions. Numerical results are presented for different conditions, such as width ratio, length ratio and the four types of boundary condition.
\end{abstract}

Keywords: Folded plate; natural vibration; vibration of continuous system

\section{Introduction}

Folded plates are widely used as a structural member on the fields of transportation, aerospace, ship and construction. Therefore, it is important to clarify and understand its vibration characteristics from a viewpoint of resonance problem in structural design.

Vibration problems of the folded plates have been studied extensively over the last few decades. Free vibration of a cantilever folded plate has been studied by the Ritz method [1]. Liu and Huang [2] showed the free vibration characteristics of a cantilever folded plate and a cylindrical curved plate by using the transfer matrix method. Free vibration of completely free folded plates considering rotational inertia has been investigated on the basis of finite element method [3]. Danial et al. [4] indicated the shock response of box-type beam based on finite element method. Free vibration of composite folded plates considering transverse shear deformation and rotational inertia has been analyzed by finite element method [5]. In addition, Nayaka et al. [6] presented transient response of composite sandwich folded plates by the use of finite element method. Most of these researches are analyzed by finite element method and detailed studies on the accuracy of solutions have been indicated. On the other hand, there are few examples of analysis showing the variations in the natural vibration characteristics due to the difference in dimensions and boundary conditions.

From the above review, this paper deals with the free vibration analysis of L-shaped folded plates by using Ritz

${ }^{*}$ Corresponding author. Tel.: +81-154-57-7294

Otanoshike-Nishi 2-32-1, Kushiro, Hokkaido, Japan, 084-0916 method and a commercial FEM (finite element method) software, SolidWorks simulation. Practically, the strain energy and the kinetic energy of the folded plate and the elastic energy of the connecting springs are evaluated, and the frequency equation is derived by applying the minimization condition to the energy functional of the folded plate. In numerical example, the accuracy and validity of solutions are shown from the convergence of solutions and the comparisons with the results in the published literature. Furthermore, the effects of dimension ratio and boundary conditions on natural vibration characteristics such as natural frequencies and vibration modes of the folded plates are illustrated and discussed.

\section{Theoretical Formulation}

\subsection{Dimensions and displacement field of the folded plate}

Figure 1 shows the dimensions and coordinate system of L-shaped folded plate composed of plate- 1 and plate- 2 . As shown in the figure, a coordinate system $0-x-y-z$ is taken and displacements in the $x, y$ and $z$ directions at arbitrary points on the folded plate are represented by $u, v$ and $w$, respectively. Also, the length and width of plate- 1 and plate- 2 are denoted by $a, b$ and $L$, and $h$ is the thickness of plates.

As shown in Fig. 2, plate-1 and plate-2 are connected by the translational springs $k_{u}, k_{v}, k_{w}$ and the rotational spring $k_{\text {rot }}$. Therefore, the relative translational and the relative angular displacements are constrained to zero on the connection line of plate- 1 and plate- 2 . 


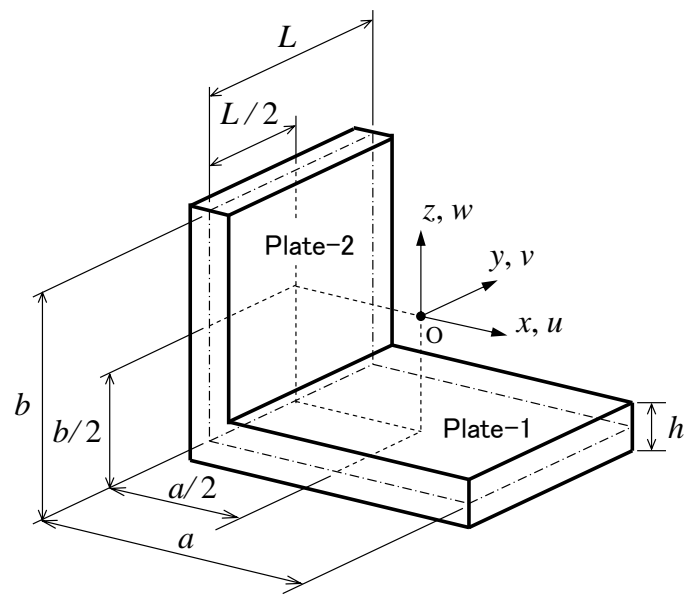

Figure 1. Dimensions and the coordinate system of L-shaped folded plate

Kirchhoff 's assumption (Line elements perpendicular to the middle plane of the plate remain straight and perpendicular to the middle plane during deformation) is applied to the bending deformation of plate- 1 and plate- 2 . In this case, displacements $u_{p}, v_{p}$ and $w_{p}(p \rightarrow 1:$ plate- 1 , $p \rightarrow 2$ : plate-2) at arbitrary points of plate- 1 and plate- 2 can be written in Eq. (1), including displacement $u_{p 0}, v_{p 0}$ and $w_{p 0}$ on the middle plane.

$$
\begin{aligned}
& u_{1}(x, y, z, t)=u_{10}(x, y, t)-\left(z+\frac{b}{2}\right) \frac{\partial w_{10}(x, y, t)}{\partial x} \\
& v_{1}(x, y, z, t)=v_{10}(x, y, t)-\left(z+\frac{b}{2}\right) \frac{\partial w_{10}(x, y, t)}{\partial y} \\
& w_{1}(x, y, z, t)=w_{10}(x, y, t) \\
& u_{2}(x, y, z, t)=u_{20}(y, z, t) \\
& v_{2}(x, y, z, t)=v_{20}(y, z, t)-\left(x+\frac{a}{2}\right) \frac{\partial u_{20}(y, z, t)}{\partial y} \\
& w_{2}(x, y, z, t)=w_{20}(y, z, t)-\left(x+\frac{a}{2}\right) \frac{\partial u_{20}(y, z, t)}{\partial z}
\end{aligned}
$$

\subsection{Potential and kinetic energies}

Assuming that the folded plate is a linear elastic body, the stress-strain relation is expressed as Eq. (2). In each subsequent equations, suffixes are replaced as $p \rightarrow 1, r \rightarrow x$, $s \rightarrow y$ for plate-1 and $p \rightarrow 2, r \rightarrow y, s \rightarrow z$ for plate-2. Also, $Q_{i}$ $j(i, j=1,2,6)$ in Eq. (2) is the stiffness coefficients determined by the modulus of longitudinal elasticity $E$ and Poisson's ratio $v$, and are given by Eq. (3).

$$
\begin{aligned}
& \left\{\begin{array}{c}
\sigma_{r p} \\
\sigma_{s p} \\
\tau_{r s p}
\end{array}\right\}=\left[\begin{array}{ccc}
Q_{11} & Q_{12} & 0 \\
Q_{12} & Q_{22} & 0 \\
0 & 0 & Q_{66}
\end{array}\right]\left\{\begin{array}{c}
\varepsilon_{r p} \\
\varepsilon_{s p} \\
\gamma_{r s p}
\end{array}\right\} \\
& Q_{11}=Q_{22}=\frac{E}{1-v^{2}}, Q_{12}=\frac{v E}{1-v^{2}}, Q_{66}=\frac{E}{2(1+v)}
\end{aligned}
$$

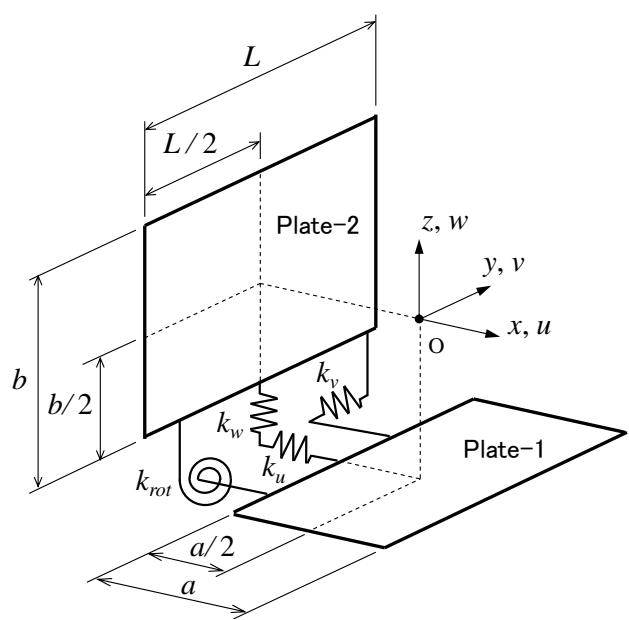

Figure 2. Connection of plate elements defined by translational and rotational springs

Considering the harmonic vibration driving at the angular frequency $\omega[\mathrm{rad} / \mathrm{s}]$ with respect to the folded plate, each displacements on the middle plane can be written as follows using the maximum amplitude $U_{p}, V_{p}$ and $W_{p}$.

$$
\begin{aligned}
& u_{p 0}(r, s, t)=U_{p}(r, s) \sin \omega t \\
& v_{p 0}(r, s, t)=V_{p}(r, s) \sin \omega t \\
& w_{p 0}(r, s, t)=W_{p}(r, s) \sin \omega t
\end{aligned}
$$

The maximum strain energy $P_{p}$ of plate-1 $(p \rightarrow 1)$ and plate-2 $(p \rightarrow 2)$ is evaluated by Eq. (5). In the equation, $[A]$ and $[D]$ are stretching and bending stiffness matrix, $\left\{\varepsilon_{p}\right\}$ and $\left\{\kappa_{p}\right\}$ are strain and curvature vector, and components of the matrices and the vectors are given as Eqs. (6) and (7).

$$
P_{p}=\frac{1}{2} \int_{s} \int_{r}\left[\left\{\varepsilon_{p}\right\}^{\mathrm{T}}[A]\left\{\varepsilon_{p}\right\}+\left\{\kappa_{p}\right\}^{\mathrm{T}}[D]\left\{\kappa_{p}\right\}\right] d r d s
$$

$$
\begin{aligned}
{[A] } & =\left[\begin{array}{ccc}
A_{11} & A_{12} & 0 \\
A_{12} & A_{22} & 0 \\
0 & 0 & A_{66}
\end{array}\right],[D]=\left[\begin{array}{ccc}
D_{11} & D_{12} & 0 \\
D_{12} & D_{22} & 0 \\
0 & 0 & D_{66}
\end{array}\right] \\
A_{i j} & =\int_{-h / 2}^{h / 2} Q_{i j} d z, D_{i j}=\int_{-h / 2}^{h / 2} Q_{i j} z^{2} d z(i, j=1,2,6)
\end{aligned}
$$

$$
\begin{aligned}
& \left\{\varepsilon_{1}\right\}=\left\{\frac{\partial U_{1}}{\partial x}, \frac{\partial V_{1}}{\partial y}, \frac{\partial U_{1}}{\partial y}+\frac{\partial V_{1}}{\partial x}\right\}^{\mathrm{T}} \\
& \left\{\kappa_{1}\right\}=\left\{-\frac{\partial^{2} W_{1}}{\partial x^{2}},-\frac{\partial^{2} W_{1}}{\partial y^{2}},-2 \frac{\partial^{2} W_{1}}{\partial x \partial y}\right\}^{\mathrm{T}} \\
& \left\{\varepsilon_{2}\right\}=\left\{\frac{\partial V_{2}}{\partial y}, \frac{\partial W_{2}}{\partial z}, \frac{\partial V_{2}}{\partial z}+\frac{\partial W_{2}}{\partial y}\right\}^{\mathrm{T}} \\
& \left\{\kappa_{2}\right\}=\left\{-\frac{\partial^{2} U_{2}}{\partial y^{2}},-\frac{\partial^{2} U_{2}}{\partial z^{2}},-2 \frac{\partial^{2} U_{2}}{\partial y \partial z}\right\}^{\mathrm{T}}
\end{aligned}
$$


Further, the maximum elastic energy $P_{u}, P_{v}$ and $P_{w}$ of the translational springs and the maximum elastic energy $P_{r o t}$ of the rotational spring can be evaluated as follows:

$$
\begin{aligned}
& P_{u}=\frac{k_{u}}{2} \int_{-L / 2}^{L / 2}\left(\left.U_{1}\right|_{x=-a / 2}-\left.U_{2}\right|_{z=-b / 2}\right)^{2} d y \\
& P_{v}=\frac{k_{v}}{2} \int_{-L / 2}^{L / 2}\left(\left.V_{1}\right|_{x=-a / 2}-\left.V_{2}\right|_{z=-b / 2}\right)^{2} d y \\
& P_{w}=\frac{k_{w}}{2} \int_{-L / 2}^{L / 2}\left(\left.W_{1}\right|_{x=-a / 2}-\left.W_{2}\right|_{z=-b / 2}\right)^{2} d y \\
& P_{r o t}=\frac{k_{r o t}}{2} \int_{-L / 2}^{L / 2}\left(\left.\frac{\partial W_{1}}{\partial x}\right|_{x=-a / 2}+\left.\frac{\partial U_{2}}{\partial z}\right|_{z=-b / 2}\right)^{2} d y
\end{aligned}
$$

where $k_{u}, k_{v}, k_{w}$ and $k_{\text {rot }}$ represent the stiffness of the translational and rotational connecting spring distributed in the $y$ direction. The maximum potential energy $P_{\max }$ of the folded plate is given by the following equation as the sum of the maximum strain energy of plate- 1 and plate- 2 and the maximum elastic energy of the connecting spring.

$$
P_{\text {max }}=P_{1}+P_{2}+P_{u}+P_{v}+P_{w}+P_{\text {rot }}
$$

On the other hand, the maximum kinetic energy $T_{p}$ of plate-1 $(p \rightarrow 1)$ and plate-2 $(p \rightarrow 2)$ is evaluated by Eq. (10) with the volume density $\rho$ of the folded plate, and the maximum kinetic energy $T_{\max }$ of the folded plate is given by Eq. (11) as the sum of the maximum kinetic energy of plate- 1 and plate- 2 .

$$
\begin{aligned}
T_{p} & =\frac{\omega^{2}}{2} \int_{s} \int_{r} \rho h\left(U_{p}^{2}+V_{p}^{2}+W_{p}^{2}\right) d r d s \\
T_{\max } & =T_{1}+T_{2}
\end{aligned}
$$

\subsection{Derivation of the frequency equation}

Displacement functions of plate-1 $(p \rightarrow 1)$ and plate-2 $(p \rightarrow 2)$ that satisfy the geometric boundary conditions at each edge of the folded plate are given by a series form as follows:

$$
\begin{aligned}
& U_{p}(r, s)=\sum_{m=0}^{I-1} \sum_{n=0}^{I-1} U_{p m n} F_{p u m n}(r, s) \\
& V_{p}(r, s)=\sum_{m=0}^{I-1} \sum_{n=0}^{I-1} V_{p m n} F_{p v m n}(r, s) \\
& W_{p}(r, s)=\sum_{m=0}^{I-1} \sum_{n=0}^{I-1} W_{p m n} F_{p w m n}(r, s)
\end{aligned}
$$

where $U_{p m n}, V_{p m n}$ and $W_{p m n}$ represent unknown coefficients. In present analysis, the four kinds of boundary edge condition Clamp-1, Clamp-2, Cantilever and Free shown in Fig. 3 are taken as a numerical example. Also, the admissible functions $F_{p d m n}(p \rightarrow 1,2$ and $d \rightarrow u, v$, $w$ ) in Eq. (12) are given as a power function from Eq. (13) to Eq. (16).

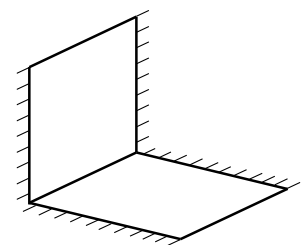

(a) Clamp-1

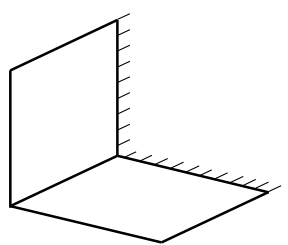

(c) Cantilever

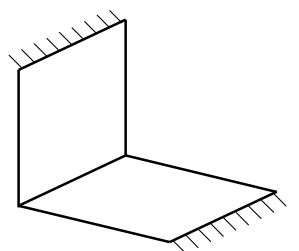

(b) Clamp-2

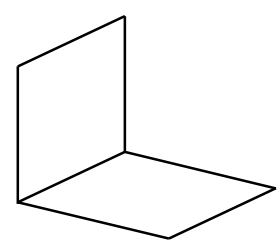

(d) Free
Figure 3. Four types of the boundary edge condition

\section{Clamp-1;}

$$
\begin{aligned}
& F_{1 u m n}(x, y)=F_{1 v m n}(x, y)=x^{m} y^{n}\left(y^{2}-L^{2} / 4\right) \\
& F_{1 w m n}(x, y)=x^{m} y^{n}\left(y^{2}-L^{2} / 4\right)^{2} \\
& F_{2 u m n}(y, z)=y^{m} z^{n}\left(y^{2}-L^{2} / 4\right)^{2} \\
& F_{2 v m n}(y, z)=F_{2 w m n}(y, z)=y^{m} z^{n}\left(y^{2}-L^{2} / 4\right)
\end{aligned}
$$

\section{Clamp-2;}

$$
\begin{aligned}
& F_{1 u m n}(x, y)=F_{1 v m n}(x, y)=x^{m} y^{n}(x-a / 2) \\
& F_{1 w m n}(x, y)=x^{m} y^{n}(x-a / 2)^{2} \\
& F_{2 u m n}(y, z)=y^{m} z^{n}(z-b / 2)^{2} \\
& F_{2 v m n}(y, z)=F_{2 w m n}(y, z)=y^{m} z^{n}(z-b / 2)
\end{aligned}
$$

\section{Cantilever;}

$$
\begin{aligned}
& F_{1 u m n}(x, y)=F_{1 v m n}(x, y)=x^{m} y^{n}(y-L / 2) \\
& F_{1 w m n}(x, y)=x^{m} y^{n}(y-L / 2)^{2} \\
& F_{2 u m n}(y, z)=y^{m} z^{n}(y-L / 2)^{2} \\
& F_{2 v m n}(y, z)=F_{2 w m n}(y, z)=y^{m} z^{n}(y-L / 2)
\end{aligned}
$$

Free;

$$
\begin{aligned}
& F_{1 u m n}(x, y)=F_{1 v m n}(x, y)=F_{1 w m n}(x, y)=x^{m} y^{n} \\
& F_{2 u m n}(y, z)=F_{2 v m n}(y, z)=F_{2 w m n}(y, z)=y^{m} z^{n}
\end{aligned}
$$

Furthermore, the displacement functions given by Eq. (12) are substituted into the maximum potential and the maximum kinetic energy described by Eqs. (9) and (11). Also, applying the minimization condition equation given by Eq. (17) to the energy functional $L=T_{\max }-P_{\max }$ derives the frequency equation as generalized eigenvalue problem given by Eq. (18).

$$
\begin{gathered}
\frac{\partial L}{\partial U_{p m n}}=\frac{\partial L}{\partial V_{p m n}}=\frac{\partial L}{\partial W_{p m n}}=0, \\
(p \rightarrow 1,2 \text { and } m, n=0,1,2, \ldots, I-1) \\
{[K]\{Q\}=\omega^{2}[M]\{Q\},} \\
\{Q\}=\left\{U_{1 m n}, V_{1 m n}, W_{1 m n}, U_{2 m n}, V_{2 m n}, W_{2 m n}\right\}^{\mathrm{T}}
\end{gathered}
$$


In Eq. (18), $[K]$ and $[M]$ are the stiffness matrix and the mass matrix, respectively. By substituting the eigenvector $\{Q\}$ into the displacement functions given by Eq. (12), the vibration mode of the folded plates can be obtained. In numerical example, the natural angular frequency $\omega[\mathrm{rad} / \mathrm{s}]$ is expressed as the nondimensional frequency $\Omega$ shown in Eq. (19).

$$
\Omega=\omega a^{2} \sqrt{\frac{\rho h}{D_{0}}} \quad\left(D_{0}=\frac{E h^{3}}{12\left(1-v^{2}\right)}\right)
$$

\section{Numerical Results and Discussions}

Based on the previous analysis, numerical study will be carried out on the free vibration characteristics of the L-shaped folded plate. In numerical calculation and FEM simulation, the longitudinal modulus of elasticity $E=207 \mathrm{GPa}$, Poisson's ratio $v=0.3$ and the volume density $\rho=7800 \mathrm{~kg} / \mathrm{m}^{3}$ are used as the material constant of the folded plate. In addition, the numerical results by SolidWorks simulation which is a commercial FEM (finite element method) software are also presented within each tables and figures.

Table 1 shows the convergence study of nondimensional frequency $\Omega$ with respect to variations of the number of terms $I \times I$ in displacement functions given by Eq. (12) and the connecting spring stiffness $k_{u}, k_{v}, k_{w}$ and $k_{r o t}$ for the folded plate having Clamp- 1 boundary condition. Nondimensional frequencies $\Omega$ converge with four significant figures in terms of the number of terms $I \times I=12 \times 12$ and spring stiffness $k_{u}=k_{v}=k_{w}=k_{\text {rot }}=10^{11}$ for each mode number. Also, nondimensional frequencies $\Omega$ are consistent with the results given by SolidWorks simulation with three significant figures. Therefore, the number of terms $I \times I=12 \times 12$ is employed in the displacement functions given by Eq. (12), and all spring stiffness are set to $10^{11}$ in present calculations.

Table 2 shows comparisons of nondimensional frequency $\Omega$ of the folded plate having Cantilever boundary condition. In the table, Irie et al. [1] show the analytical solutions by the Ritz method, Liu and Huang [2] and Haldar and Sheikh [5] give results by the finite element method. In particular, the transverse shear deformation is taken into account on the bending deformation of the folded plate in [5].

Table 3 shows comparisons of the nondimensional frequency $\Omega$ with respect to the folded plate with Free boundary condition. Allman [3] gives experiment results and Nayak et al. [6] present analytical solutions by the finite element method considering transverse shear deformation and rotational inertia in free vibration analysis of the folded plates.

In Tables 2 and 3, the present solutions are in good agreement with the results by the references, and it is shown that connecting conditions of translation and rotation between plate- 1 and plate- 2 are sufficiently satisfied by using the connecting spring. Also, the accuracy and validity of the present solutions are verified through comparing with results from references.
Table 1. Convergence study of nondimensional frequencies $\Omega$ [ $a / b=1, L / a=1, h / a=0.01$, Clamp- 1$]$

\begin{tabular}{|c|c|c|c|c|c|}
\hline \multirow{2}{*}{$\begin{array}{l}\text { Mode } \\
\text { No. }\end{array}$} & \multirow{2}{*}{$\begin{array}{l}\text { Num.of } \\
\text { terms } I \times I\end{array}$} & \multicolumn{4}{|c|}{ Spring stiffness $\left(k_{u}, k_{v}, k_{w}, k_{r o t}\right)$} \\
\hline & & $10^{8}$ & $10^{9}$ & $10^{10}$ & $\underline{10^{11}}$ \\
\hline \multirow{5}{*}{1} & $9 \times 9$ & 23.362 & 23.373 & 23.374 & 23.374 \\
\hline & $10 \times 10$ & 23.360 & 23.371 & 23.373 & 23.373 \\
\hline & $11 \times 11$ & 23.358 & 23.369 & 23.371 & 23.371 \\
\hline & $\underline{12 \times 12}$ & 23.358 & 23.369 & 23.370 & 23.370 \\
\hline & FEM & & & & 23.366 \\
\hline \multirow{5}{*}{2} & $9 \times 9$ & 23.903 & 23.922 & 23.924 & 23.925 \\
\hline & $10 \times 10$ & 23.901 & 23.921 & 23.923 & 23.923 \\
\hline & $11 \times 11$ & 23.899 & 23.918 & 23.921 & 23.921 \\
\hline & $12 \times 12$ & 23.898 & 23.918 & 23.920 & $\underline{23.920}$ \\
\hline & FEM & & & & 23.915 \\
\hline \multirow{5}{*}{3} & $9 \times 9$ & 35.415 & 35.554 & 35.570 & 35.572 \\
\hline & $10 \times 10$ & 35.413 & 35.552 & 35.568 & 35.570 \\
\hline & $11 \times 11$ & 35.411 & 35.550 & 35.566 & 35.568 \\
\hline & $\underline{12 \times 12}$ & 35.411 & 35.549 & 35.566 & $\underline{35.568}$ \\
\hline & FEM & & & & 35.548 \\
\hline \multirow{5}{*}{4} & $9 \times 9$ & 39.618 & 39.955 & 39.990 & 39.993 \\
\hline & $10 \times 10$ & 39.615 & 39.952 & 39.987 & 39.990 \\
\hline & $11 \times 11$ & 39.614 & 39.951 & 39.985 & 39.989 \\
\hline & $\underline{12 \times 12}$ & 39.613 & 39.950 & 39.984 & $\underline{39.988}$ \\
\hline & FEM & & & & 39.962 \\
\hline \multirow{5}{*}{5} & $9 \times 9$ & 62.845 & 62.881 & 62.886 & 62.886 \\
\hline & $10 \times 10$ & 62.838 & 62.875 & 62.880 & 62.880 \\
\hline & $11 \times 11$ & 62.836 & 62.873 & 62.877 & 62.878 \\
\hline & $\underline{12 \times 12}$ & 62.833 & 62.870 & 62.874 & $\underline{62.875}$ \\
\hline & FEM & & & & 62.852 \\
\hline
\end{tabular}

Table 2. Comparisons of nondimensional frequencies $\Omega$ [ $a / b=1, L / a=2, h / a=0.04$, Cantilever ]

\begin{tabular}{lccccc}
\hline \multirow{2}{*}{ Source } & \multicolumn{5}{c}{ Mode No. } \\
\cline { 2 - 6 } & 1 & 2 & 3 & 4 & 5 \\
\hline \hline Present (Ritz) & 2.12 & 4.21 & 7.727 & 9.050 & 14.95 \\
Present (FEM) & 2.12 & 4.21 & 7.722 & 9.042 & 14.95 \\
Ritz method [1] & 2.13 & 4.23 & 7.768 & 9.098 & 15.47 \\
FEM [2] & 2.13 & 4.20 & 7.734 & 9.024 & 15.41 \\
FEM [5] & 2.11 & 4.16 & 7.734 & 8.985 & 14.80 \\
\hline
\end{tabular}

Table 3. Comparisons of nondimensional frequencies $\Omega$ [ $a=0.28 \mathrm{~m}, b=0.428 \mathrm{~m}, L=0.215 \mathrm{~m}, h=0.00121 \mathrm{~m}$, $\rho=7850 \mathrm{~kg} / \mathrm{m}^{3}$, Free ]

\begin{tabular}{lcccc}
\hline \multirow{2}{*}{ Source } & \multicolumn{4}{c}{ Mode No. } \\
\cline { 2 - 5 } & 1 & 2 & 3 & 4 \\
\hline \hline Present (Ritz) & 2.795 & 6.464 & 8.505 & 9.911 \\
Present (FEM) & 2.794 & 6.462 & 8.503 & 9.907 \\
Exp. [3] & 2.748 & 6.612 & 8.446 & 9.547 \\
FEM [6] & 2.832 & 6.468 & 8.630 & 9.937 \\
\hline
\end{tabular}

Figures 4 and 5 show vibration modes with respect to variation in the length ratios $a / b$ for the folded plates having Clamp-1 and Free boundary conditions, respectively. Nondimensional frequency $\Omega_{\text {Ritz }}$ and $\Omega_{\text {FEM }}$ by present analysis and SolidWorks simulation are shown under each vibration modes. The folded plates having Clamp- 1 with the constraint of the edge shows higher $\Omega$ 
than the plates having Free boundary condition. In any of the boundary conditions, nondimensional frequencies $\Omega$ increase as the length ratio $a / b$ increases. Also, vibration mode shape of the folded plate has a beam type mode when $a / b=0.1$, and a plate type mode when $a / b=10$.

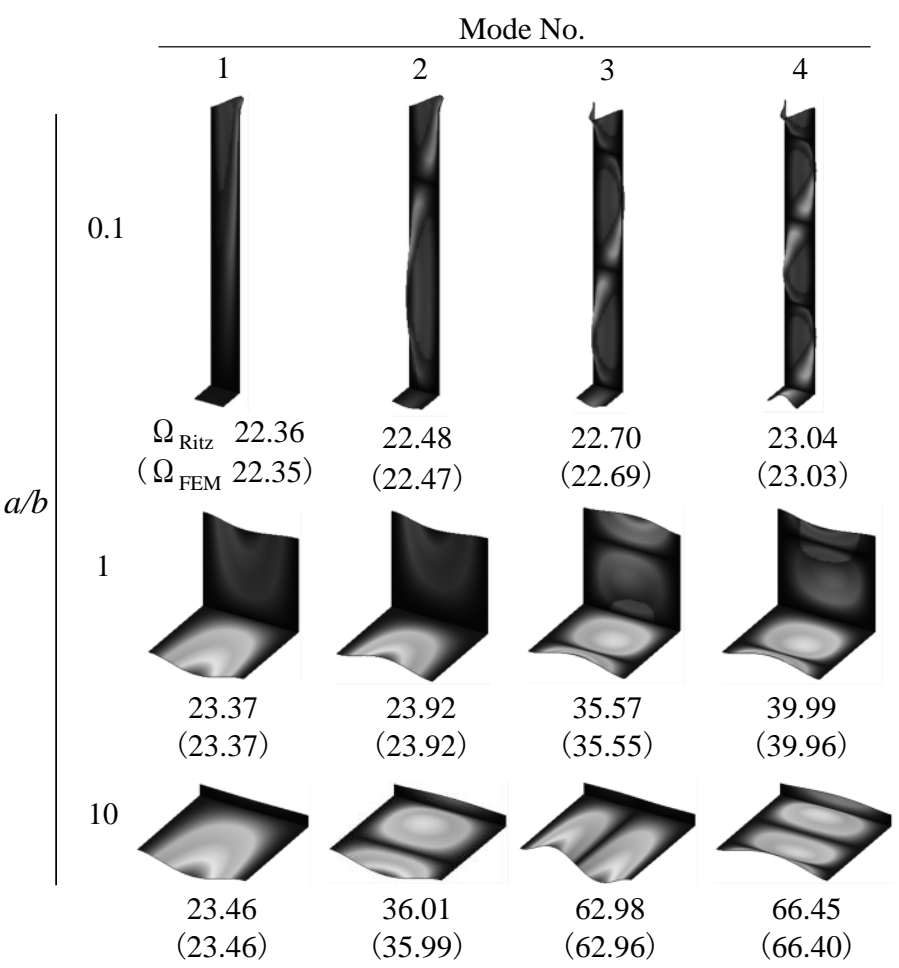

Figure 4. Vibration modes of L-shaped folded plates [ $L / a=1, h / a=0.01$, Clamp -1 ]

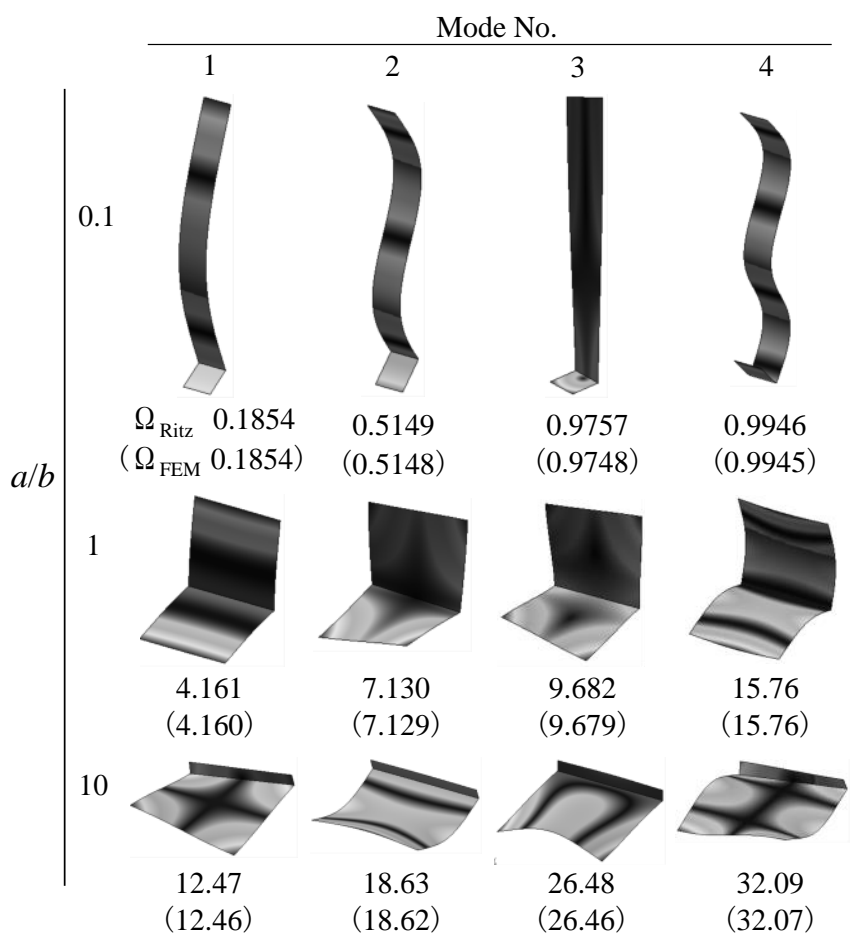

Figure 5. Vibration modes of L-shaped folded plates [ $L / a=1, h / a=0.01$, Free ]

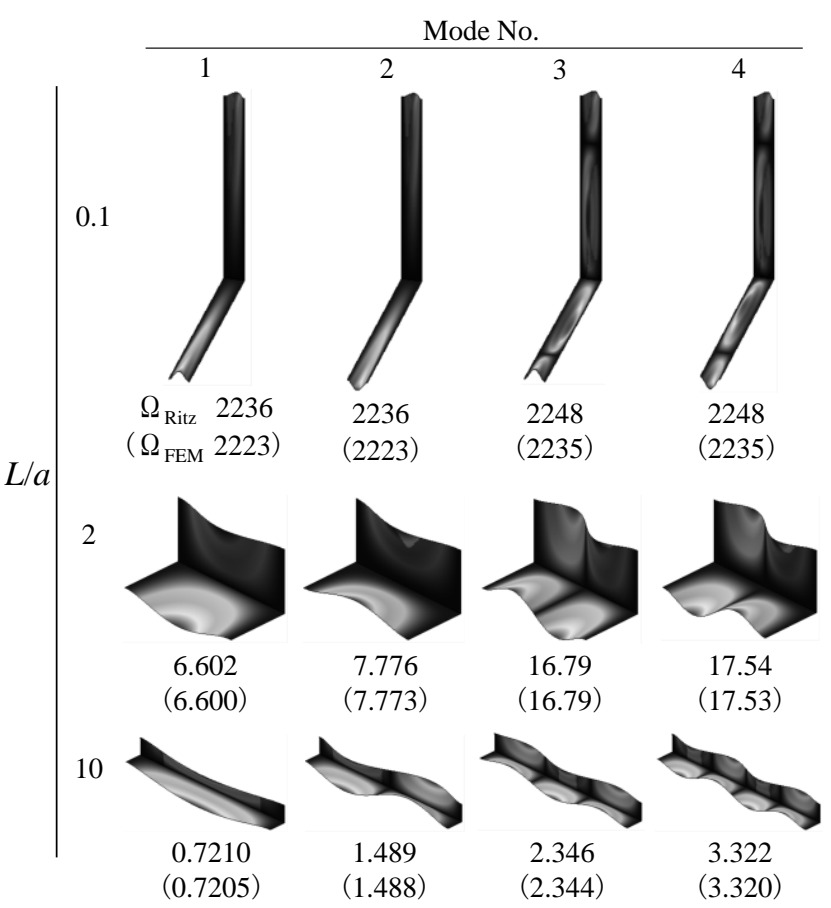

Figure 6. Vibration modes of L-shaped folded plates $[a / b=1, h / a=0.01$, Clamp-1 ]

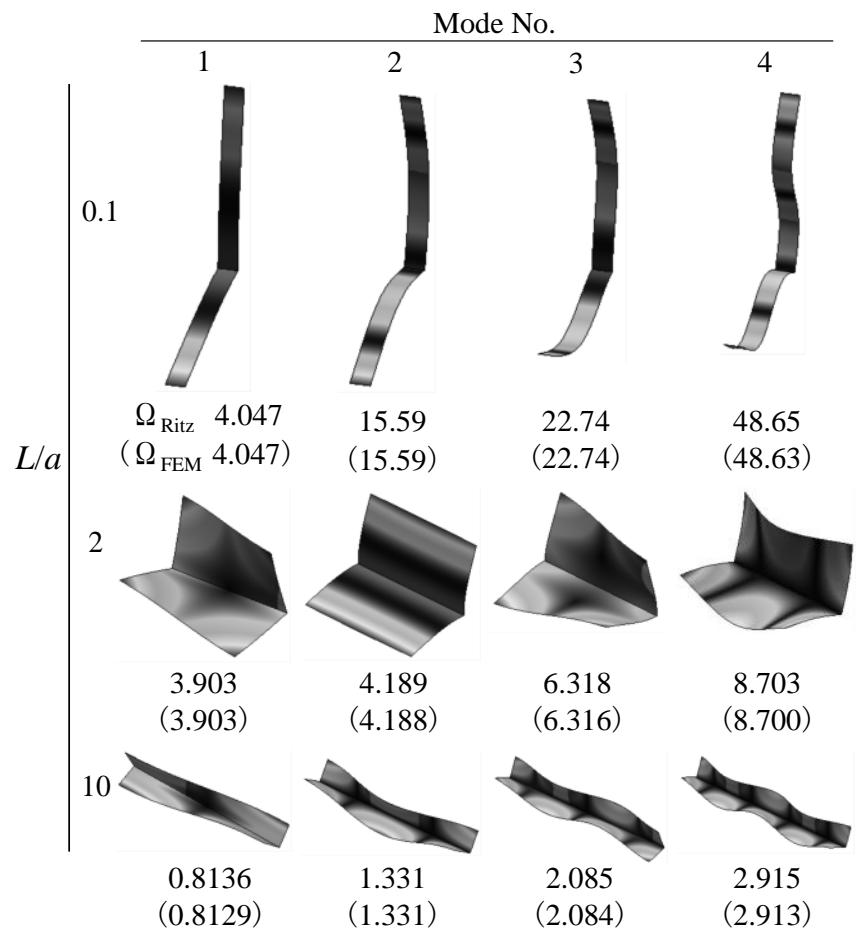

Figure 7. Vibration modes of L-shaped folded plates [ $a / b=1, h / a=0.01$, Free ]

Figsures 6 and 7 give vibration modes with respect to variation versus the width ratio $L / a$ for the folded plates with Clamp-1 and Free boundary conditions. In the both of the boundary conditions, nondimensional frequencies $\Omega$ decrease as the width ratio $L / a$ increases. The reduction rate of $\Omega$ with respect to the increase in the width ratio $L / a$ 


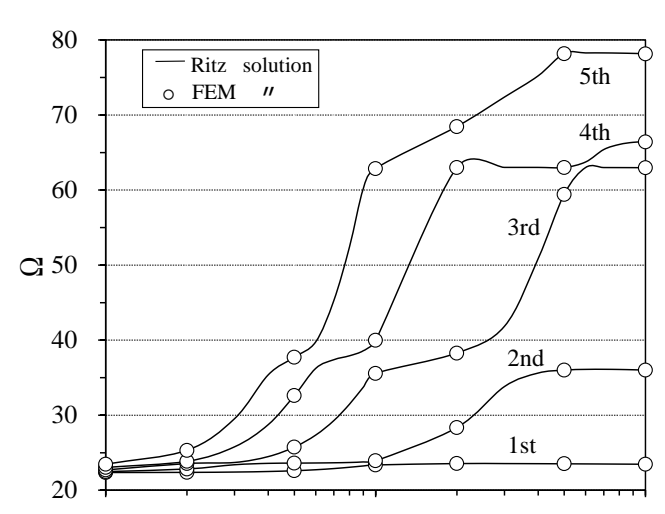

(a) Clamp-1

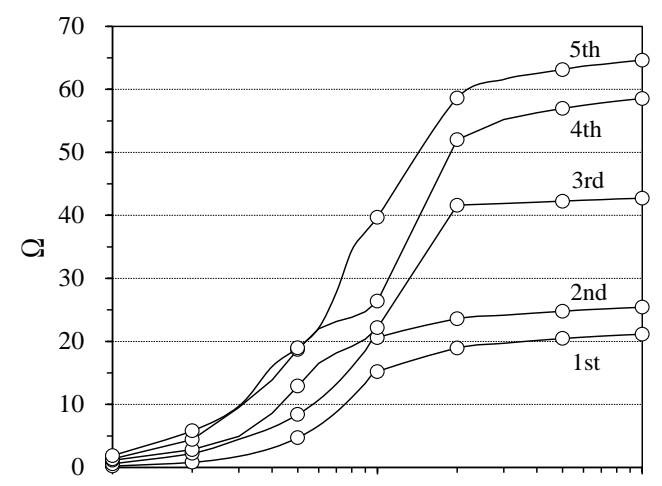

(b) Clamp-2

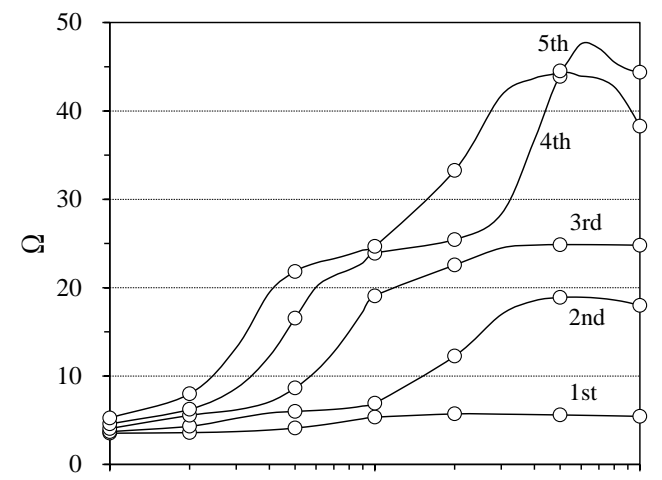

(c) Cantilever

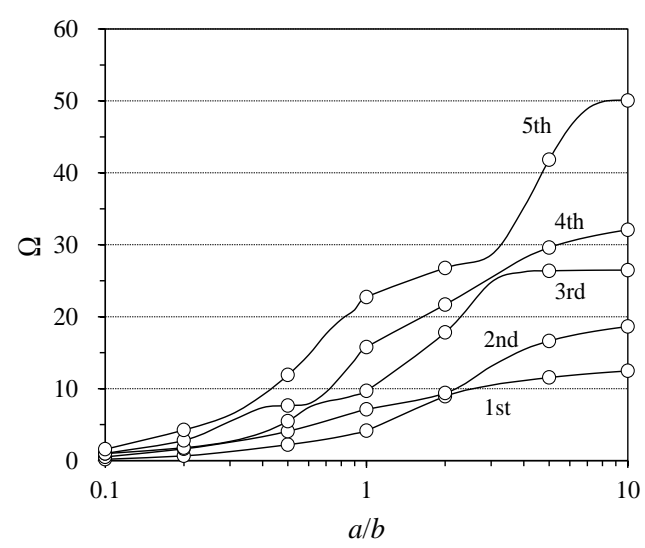

(d) Free

Figure 8. Variations of nondimensional frequencies $\Omega$ versus the length ratio for $\mathrm{L}$-shaped folded plates [ $L / a=1, h / a=0.01$ ]

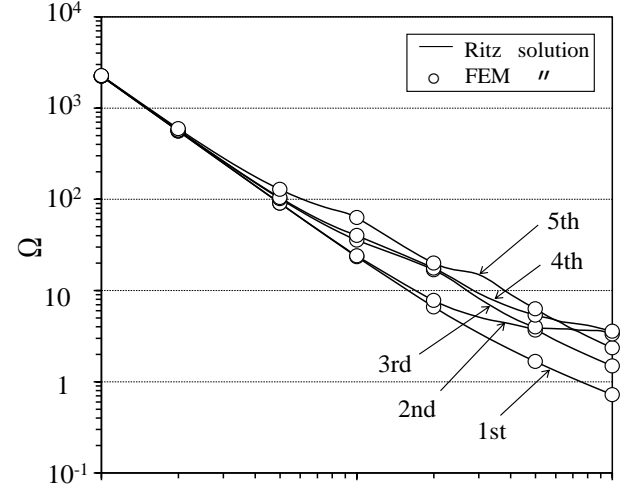

(a) Clamp-1

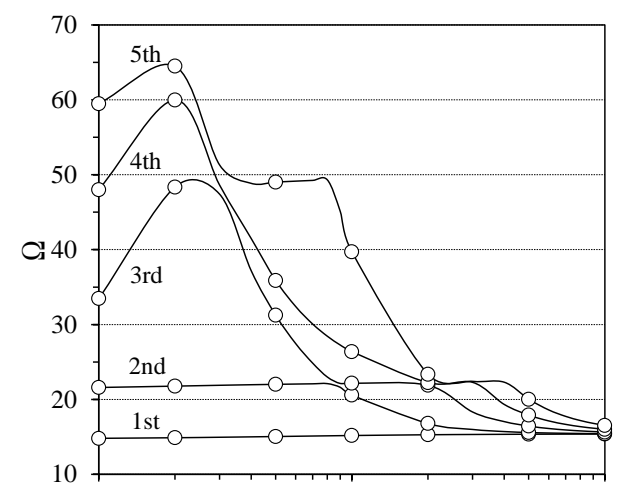

(b) Clamp-2

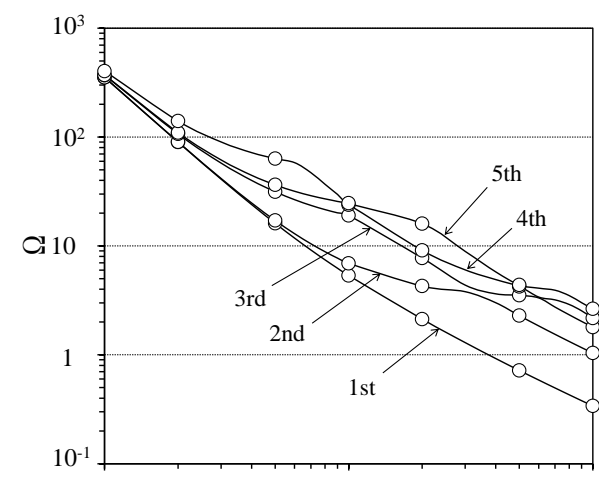

(c) Cantilever

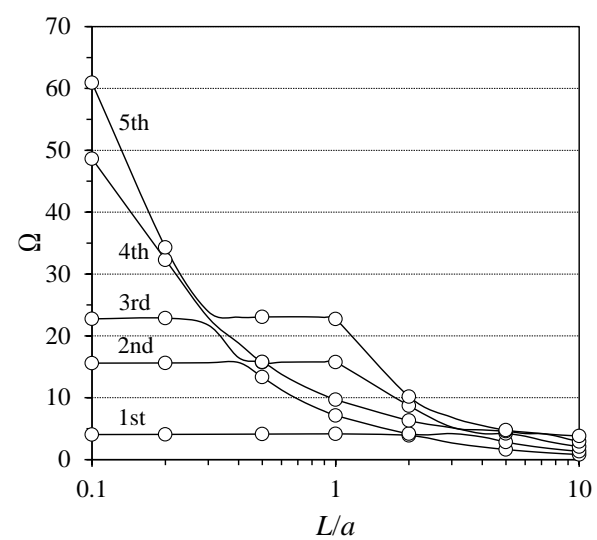

(d) Free

Figure 9. Variations of nondimensional frequencies $\Omega$ versus the width ratio for $\mathrm{L}$-shaped folded plates [ $a / b=1, h / a=0.01$ ] 
is larger in the folded plate having Clamp- 1 than in the folded plate having Free boundary condition without constraint on the edge side. The folded plate has the beam type vibration modes when $L / a=0.1$ and 10 , and shows the plate type vibration modes when $L / a=2$, because plate- 1 and plate- 2 become the flat plates in the case of $L / a=2$.

Figure 8 shows the variations of the nondimensional frequency $\Omega$ with respect to the length ratio $a / b$ for the folded plates with Clamp-1, Clamp-2, Cantilever and Free boundary conditions. In the figure, the solid line shows the present solution, and the small circles show the solutions by SolidWorks simulation. The folded plate having Clamp-1, which has constraint on four sides gives high $\Omega$. Also, in any boundary condition, $\Omega$ show an increasing tendency as the length ratio $a / b$ increases. In addition, veering points which frequency curves of different modes approach each other are observed in 3rd and 4th mode of Clamp-1, 2nd and 3rd mode of Clamp-2, 4th and 5th mode of Cantilever, 1st and 2nd mode of Free.

Figure 9 shows the variations of the nondimensional frequency $\Omega$ with respect to the width ratio $L / a$ for the folded plates with the four kinds of boundary condition Clamp-1, Clamp-2, Cantilever and Free. On the folded plates having Clamp- 1 and Cantilever, high $\Omega$ is given in the range of the width ratio $L / a<1$. Also, in any boundary condition, $\Omega$ show a decreasing tendency as the width ratio $L / a$ increases. On the other hand, the variations in the fundamental frequency with respect to the width ratio $L / a$ is scarce in Clamp-2 and Free where there is no constraint on the sides.

\section{Concluding Remarks}

In this study, the free vibration analysis of the Lshaped folded plates has been formulated by applying the Ritz method. Several perceptions obtained through discussion considering numerical examples are as follows.

On natural frequencies of the L-shaped folded plates, accuracy and validity of present solutions were verified from the comparisons with results by FEM software and literature. Also, it was shown that connecting conditions of translation and rotation between two plates are satisfied by using the connecting spring. In addition, vibration modes of the folded plates present a beam type and a plate type shape according to the length ratio and the width ratio. Regarding the variations of frequency curves, for each boundary condition, natural frequencies show an increasing as the length ratio increases and a decreasing as the width ratio increases.

It is expected that present numerical results are useful for the optimization design on vibration problems of the folded plates.

\section{References}

[1] Irie T, Yamada G, Kobayashi Y. Free vibration of a cantilever folded plate. J Acoust Soc Am 1984;6:1743-48.

[2] Liu WH, Huang CC. Vibration analysis of folded plates. J Sound Vib 1992;1:123-37

[3] Allman DJ. Implementation of a flat facet shell finite element for applications in structural dynamics. Comput Struct 1996;4:657-63.

[4] Danial AN, Doyle JF, Rizzi SA. Dynamic analysis of folded plate structures. Trans ASME J Vib Acoust 1996;118:591-98.

[5] Haldar S, Sheikh AH. Free vibration analysis of isotropic and composite folded plates using a shear flexible element. Finite Elem Anal Des 2005; 42:208-26.

[6] Nayak AK, Shenoi RA, Blake JIR. A study of transient response of initially stressed composite sandwich folded plates. Compos B Eng 2013;44:67-76. 